\title{
Monitoring Wireless Sensor Networks Using a Model-Aided Approach
}

\author{
Chongqing Zhang, Minglu Li, Min-You Wu, and Wenzhe Zhang \\ Department of Computer Science and Engineering, \\ Shanghai Jiaotong University, Shanghai, China \\ zhangchongqing@sjtu.edu.cn
}

\begin{abstract}
A wireless sensor network may consist of a large number of small, battery-powered, wireless sensor nodes and works in an unattended way. In order to manage the sensor network and collect data from the network efficiently, we need to know the state of the WSN. In this paper, we propose a model-aided approach to support the monitoring of the state of WSNs. In this approach, models are created on base station to support the monitoring of the network, and mobile agents are injected into the network to collect state information. Experimental results show the effectiveness of our approach.
\end{abstract}

\section{Introduction}

A WSN may consist of a large number of low-power sensors and/or actuators with limited sensing, processing, and wireless communication capabilities. After being deployed, those nodes self-organize into an integral network and work in an unattended way. In order to work properly and efficiently, applications need to reconfigure and adapt themselves based on the state information of the WSN.

For example, Database technology has been adopted by many works [1, 2] as an effective way for managing the data of WSNs. Users generally interact with a WSN database by queries and responses. In order to work out query plans of high efficiency, the base station needs to know overall state of the network so as to parse and optimize the queries submitted by users. Yet these works didn't discuss how to obtain the state knowledge of WSNs. Knowing the state of a WSN can also facilitate the network management work. By knowing the state of the WSN, users can get the knowledge of the health condition of the network and thus network management works, e.g. incremental deployment of sensor nodes, can be done efficiently.

In [3], Jerry Zhao, et al, proposed an approach called Sensor Network Tomography for monitoring the state of WSN. Instead of collecting detailed state information from each individual sensor node and then process centrally, their approach builds abstracted scans of sensor network health by combining local scans piecewise on their way towards a collecting point. And in [4], they implemented a residual energy scan (eScan) which approximately describes the remaining energy distribution within a WSN. By adopting aggregation techniques, the communication cost of Sensor Network Tomography can be reduced. Budhaditya Deb, et al, introduced in [5] a topology discovery algorithm for WSNs. The algorithm uses only a set of distinguished nodes to reply back to the topology 
discovery probes. And by using the retrieved information, approximate topology of the network can be constructed.

In this paper, we proposed a novel model-based approach for monitoring state of WSNs. The main idea of this approach lies in the models that depict the state of WSNs and how the state changes. Those models take full advantage of the rules of how the state of WSNs changes, the relations or correlations between the attributes of sensor nodes or sensor groups. By using proper models to predict the state of WSNs, this approach can overcome the long delay and probe effect introduced by the approaches mentioned above; and the energy cost can also be reduced significantly.

The rest of this paper is organized as follows. In section II, We give the WSN model on which our research are based and present an overview of our approach. In section III, the WSNs state monitoring architecture and state information collecting methods are presented. Experimental results are presented in section IV to show the effectiveness of our approach. We conclude in section V.

\section{WSN Model and Overview of Approach}

\subsection{Wireless Sensor Network Model}

Without loss of generality, the WSNs model used in this paper is based on following assumptions:

1) A WSN is composed of a base station and large number of nodes scattered on a plane. Each node has a unique identifier. Nodes don't have to be homogeneous.

2) Base station and nodes can move at a relatively low speed.

3) Software environments that support mobile agents are installed on base station and sensor nodes [6].

\subsection{Overview of Approach}

The change of the state of a WSN follows some rules, and there are relations and correlations between different states of the WSN or sensor node. The rules and relations enable us to estimate or predict the state of the WSN by corresponding techniques.

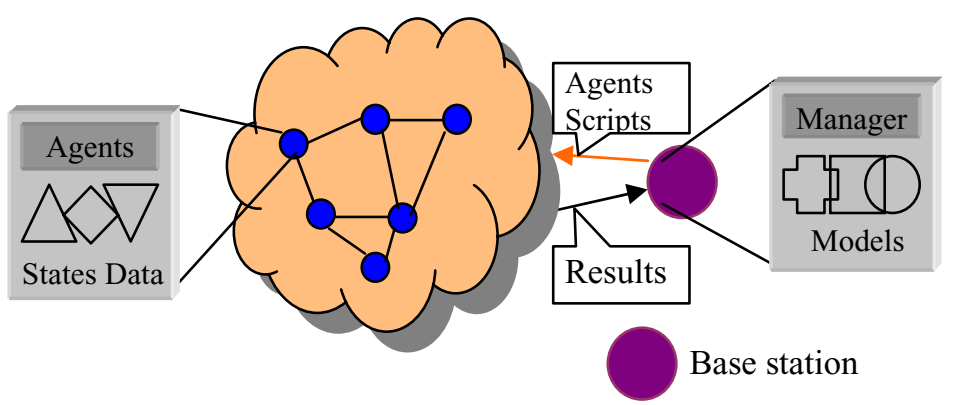

Fig. 1. Overview of Our Approach 
To make full use of those rules and relations, we propose an approach for monitoring the state of WSNs. Fig. 1 gives an overview of our approach. Models depicting the state of the WSN are created on the base station, and there is a manager that is responsible for the management work of the models. The models not only can be used to support query processing and network management work, but also can be used to collect state information of the WSN from sensor nodes. The state information collecting work can be done using mobile agents. The models manager can use many strategies to collect state information of the WSN. For example, when the models can't reflect the real state of the WSN faithfully, the models manager will issue corresponding agents to collect needed state information.

\section{Monitoring Architecture}

Monitoring the state of a WSN is a challenging work and deserves being studied carefully. The monitoring system should introduce minimal impact on network lifetime, scale with network size; yet preserve the fidelity of the overall picture of the WSN.

\subsection{Monitoring Architecture}

As the reply to above-mentioned challenges, we propose two WSN state monitoring architectures to facilitate the monitoring of flat or hierarchical WSNs [7]. The architectures are illustrated by Fig. 1 and Fig. 2 respectively. As for a flat WSN, on the base station side, there is a models manager in charge of the management work of the models. The models manager also issues agents with different triggering conditions and injects them into the network to collect state information from sensor nodes. On sensor nodes side, agents issued by the base station monitors the state changes of the node. When the conditions of an agent are met, it sends the state information back to the base station.

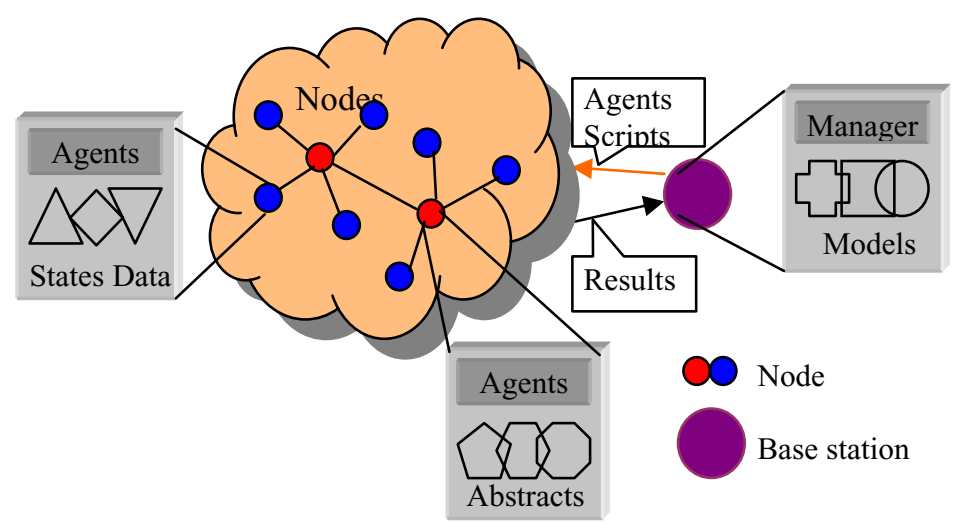

Fig. 2. State Monitoring Architecture for Hierarchical WSNs 
In a hierarchical WSN, nodes are grouped into clusters and each cluster has a cluster head. As the architecture for flat WSNs, the models manager of base station also manages models and issues agents and injected them into the network, yet these agents are only issued for cluster heads. A cluster head also issues agents and sends them to the nodes belonging to the cluster. The agents that reside in the nodes belonging to the cluster send state information to the cluster head, and then the state information is aggregated into abstracts depicting the state of the cluster. And then the abstracts are reported to the base station. Note that the cluster head of a cluster may change, so agents should be able to move from old cluster head to new cluster head.

\subsection{Using Agents to Collect State Information}

As figure 3 depicts, the state information collecting approach has following functional steps among which steps 2, 4, 5, 6 run on base station, steps 3, 7 run on nodes, and step 1 runs on both base station and nodes.

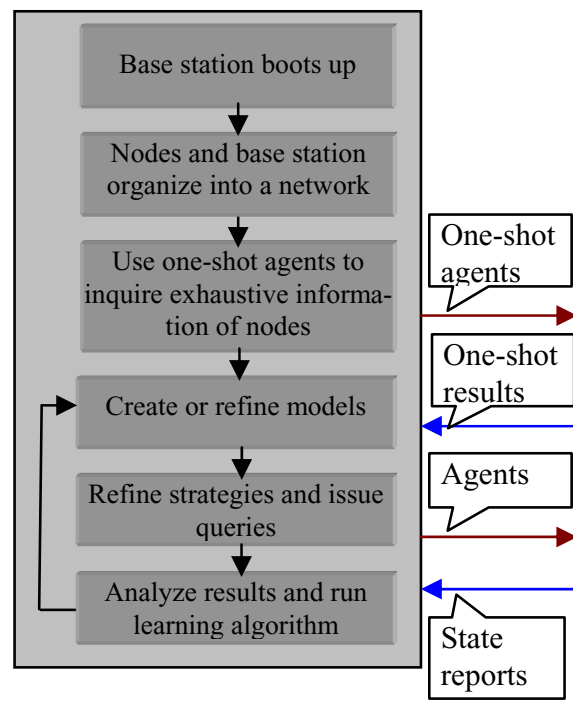

Base station side

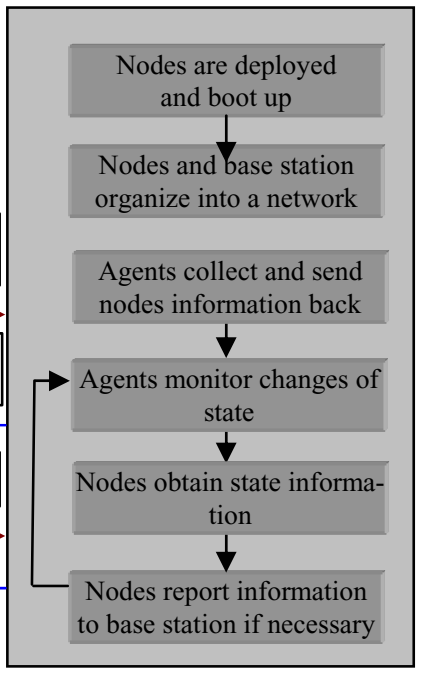

Nodes side

Fig. 3. Collecting State Information from Nodes

\section{Experimental Results}

As Fig. 4, one of the simulation scenes, shows, the WSN model consists of 200 sensor nodes scattered on a $300 \mathrm{~m} \times 300 \mathrm{~m}$ square area. The base station is located at the border of the simulation area. All nodes have same transmission ranges of 40 meters. 10 nodes can move along a straight line and at a relatively low speed less than $0.2 \mathrm{~m} / \mathrm{s}$. The initial energy of a sensor node is 5 joules, and the energy of the base station is infinite. The energy needed for a sensor node to receive and transmit a packet is 

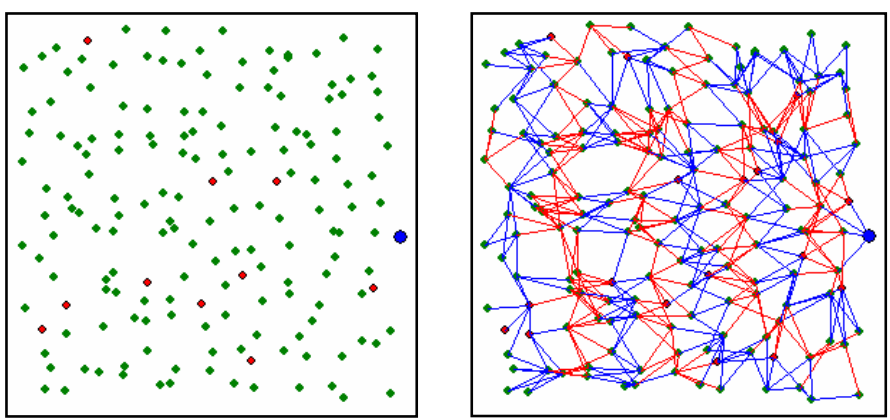

Fig. 4. An Example of WSNs Used in Experiments

$2 \times 10^{-6}$ joule and $1 \times 10^{-5}$ joule respectively. The power for a mobile node to move is $5 \times 10^{-5} \mathrm{w}$. For simplicity, an agent and the state information of a node are all transmitted as a data packet.

We compare five metadata management approaches: 1) NMLQ is not model-aided, and agents collect and report state information to the base station periodically; 2) NMR is not model-aided, and agents only report state information to the base station as significant changes happen; 3) MLQ is model-aided, and agents collect and report state information to the base station periodically; 4) MR is model-aided, agents only report state information to the base station as significant changes happen; 5) MOQ is model-aided, and state information is collected only when the confidence of a model is less than corresponding threshold.

We use two metrics, energy cost and fidelity to evaluate different approaches in our simulation. We simply calculate the energy cost by taking count of the data packets used for transmitting state information and agents. The higher the value is; the worse is the performance. Fidelity can be evaluated by the errors between the state value given by base station and the real state of the WSN. We use the error of the number of packets nodes generated and relayed to evaluate the fidelity of all approaches.

Fig. 5 compares the absolute energy cost of all approaches in one minute in detail. It can be seen that the energy cost by MOQ is less than other four approaches.

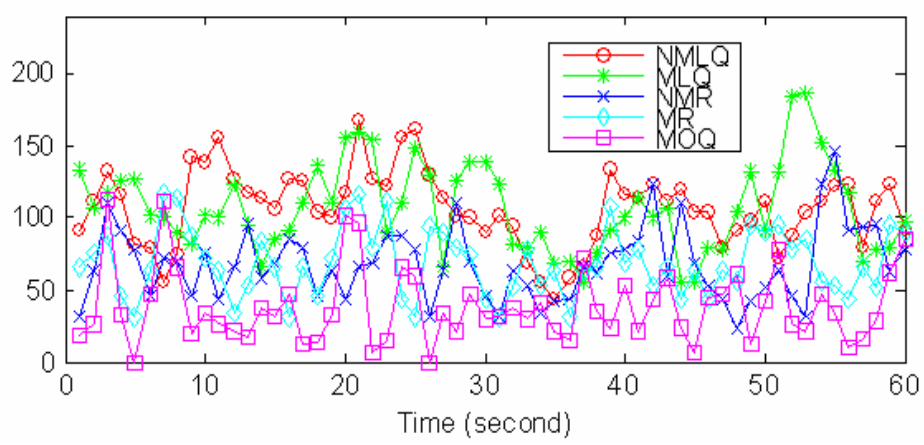

Fig. 5. Energy Consumption of All Approaches 


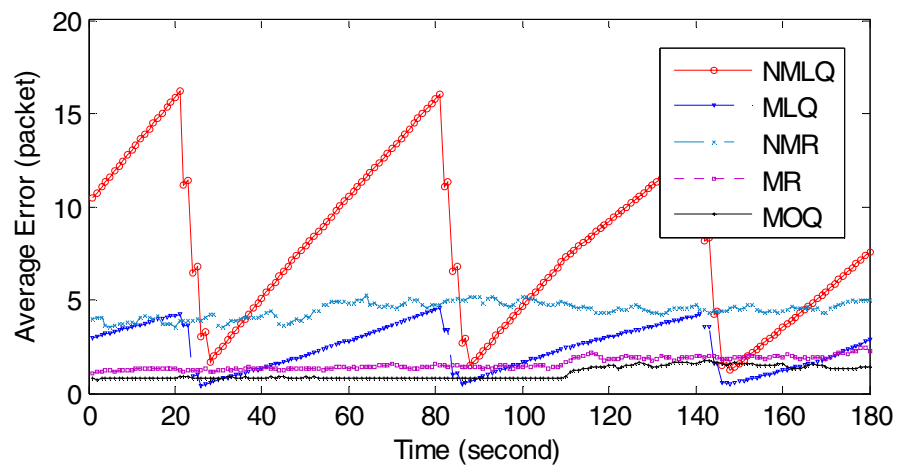

Fig. 6. Errors Comparison between All Approaches

Fig. 6 compares the errors of five approaches in 3 minutes. From the figure, the performances of model-aided MLQ and MR outscore the performances of their corresponding non-model-aided counterparts: NMLQ and NMR. Among five approaches, helped by models, MOQ consumes least energy and has the best precision.

\section{Conclusion}

In this paper, we propose a novel model-aided approach to support the monitoring of WSN state. This approach takes advantage of the rules of how the state of WSNs changes and relations or correlations between the attributes of nodes or nodes groups. By using proper models to predict the state of WSNs, this approach can overcome the long delay and probe effect introduced by the approaches mentioned above; and the energy cost can also be reduced significantly. Experimental results show the effectiveness of our approach.

\section{References}

1. Yao Y, Gehrke J. "The cougar approach to in-network query processing in sensor networks". SIGMOD Record, 2002,31(3):918.

2. Sam Madden, Joe Hellerstein, and Wei Hong. "TinyDB: In-Network Query Processing in TinyOS”. Version 0.4, September 2003.

3. J. Zhao, R. Govindan, and D. Estrin. "Sensor Network Tomography: Monitoring Wireless Sensor Networks"; Student Research Poster. ACM SIGCOMM 2001.

4. J. Zhao, R. Govindan, and D. Estrin. Residual energy scans for monitoring wireless sensor networks. IEEE WCNC, 2002.

5. S. B. B. Deb and B. Nath, "A Topology Discovery Algorithm for Sensor Networks with Applications to Network Management," Tech. rep. DCSTR-441, Dept. of Comp. Sci., Rutgers Univ., May 2002.

6. Lang Tong; Qing Zhao; Adireddy, S. Sensor networks with mobile agents. MILCOM 2003.

7. Lotfinezhad, M.; Ben Liang. Energy efficient clustering in sensor networks with mobile agents. WCNC 2005. 